Check for updates

Cite this: RSC Adv., 2020, 10, 16659

\title{
Exploring the binding mode and thermodynamics of inverse agonists against estrogen-related receptor alpha†
}

\begin{abstract}
Konda Reddy Karnati, (D) ${ }^{a}$ Yixuan Wang (D) *a and Yongli Du ${ }^{b}$
Since estrogen-related receptor alpha $(E R R \alpha)$, one of three estrogen-related receptors, displays constitutively active transcriptional activities and important implications in both physiological and pathological processes of breast cancers, ERR $\alpha$ was recently recognized as a new target to fight breast cancers, and regulating the activity of ERR $\alpha$ with inverse agonists has thus become a promising new therapeutic strategy. A few inverse agonists cyclohexylmethyl-(1-p-tolyl- $1 \mathrm{H}$-indol-3-ylmethyl)-amine (compound 1), thiadiazoacrylamide (XCT790), and 1-(2,5-diethoxy-benzyl)-3-phenyl-area analogues (compounds 2 and 3) were reported to be capable of targeting ERR $\alpha$. However, the detailed mechanism by which the inverse agonists deactivate ERR $\alpha$ remains unclear, especially in the aspects of quantitative binding and hot spot residues. Therefore, to gain insights into the interaction modes between inverse agonists and ERR $\alpha$ ligand binding domain, all-atom molecular dynamics (MD) simulations were firstly carried out for the complexes of inverse agonists and ERR $\alpha$. The binding free energies were then calculated with MM-PBSA method to quantitatively discuss the binding of the inverse agonists with ERR $\alpha$. The binding affinities were finally decomposed to per-residue contributions to identify the hot spot residues as well as assess their role in the binding mechanism. MD simulations show that the inverse agonists stretch downwards into the ERR $\alpha$ ligand binding pocket (LBP) formed by $\mathrm{H} 3$ and $\mathrm{H} 11$ helices, and upon the binding $\mathrm{H} 12$ adopts a well-defined position in the coactivator groove, where PGC-1 $\alpha$ binds to ERR $\alpha$. Binding energy analysis indicates that compound 3 and XCT790 bind more tightly to ERR $\alpha$ than compounds 1 and 2, and the energy difference mainly results from the contribution of van der Waals interaction. Both binding mode analysis and affinity decomposition per-residue indicate that compound 1, XCT790, and compound 3 have similar binding spectra to $E R R \alpha$, primarily interacting with the residues of $\mathrm{H} 3, \mathrm{H} 5, \mathrm{H} 6 / \mathrm{H} 7$ loop, and $\mathrm{H} 11$ helix, while compound 2 lacks a significant interaction with the $\mathrm{H} 5$ region. The hot spot residues significantly binding to the three inverse agonists in common include Leu324, Phe328, Phe382, Leu398, Phe495, and Leu500. It is essential for an effective inverse agonist to strongly bind with the aromatic ring cluster consisting of Phe328(H3), Phe495(H11), and Phe382(H5/H6 loop) as well as Leu500.
\end{abstract}

rsc.li/rsc-advances

Received 19th December 2019 Accepted 31st March 2020

DOI: 10.1039/c9ra10697a

\section{Introduction}

Estrogen-related receptor $\alpha(\mathrm{ERR} \alpha)$ is one of three nuclear hormone receptors, $\operatorname{ERR} \alpha, \operatorname{ERR} \beta$, and $\operatorname{ERR} \gamma .{ }^{\mathbf{1 , 2}}$ It shares high homology to estrogen receptor $\alpha(\mathrm{ER} \alpha)$ at the DNA-binding domain, and recognizes ERR $\alpha$ response elements $\left(5^{\prime}\right.$ TNAAGGTCA $\left.^{\prime}\right)$, and modulates the action of ER $\alpha .{ }^{3,4}$ Unlike ERs, ERRs are not activated by known natural estrogens, and are therefore classified as orphan receptors. ${ }^{5,6}$ The activity of ERR $\alpha$

${ }^{a}$ Department of Chemistry and Forensic Science, Albany State University, Albany, GA 31705, USA. E-mail: yixuan.wang@asurams.edu

${ }^{b}$ School of Chemical and Pharmaceutical Engineering, Qilu University of Technology (Shandong Academy of Sciences), Jinan, Shandong 250353, China

$\dagger$ Electronic supplementary information (ESI) available. See DOI: 10.1039/c9ra10697a is primarily controlled by its level of expression and interaction with coactivators; in particular, its most common interaction is with the peroxisome proliferator-activated receptor $\gamma$ coactivator $1 \alpha(\mathrm{PGC}-1 \alpha){ }^{7,8}$ The PGC-1 $\alpha / \mathrm{ERR} \alpha$ axis has thus been implicated in controlling the expression of metabolic gene networks and mitochondrial biogenesis. ${ }^{9-11}$ ERR $\alpha$ is expressed in a wide variety of tissues and its high expression correlates with poor prognosis in breast, colon, and ovarian cancers. ${ }^{\mathbf{1 2}}$ Importantly, over-expression of $\mathrm{ERR} \alpha$ is linked to poor clinical outcome for breast cancer. ${ }^{6}$ Therefore, regulating the activity of ERR $\alpha$ with inverse agonists has been considered as a promising new therapeutic strategy for the treatment of breast cancer, particularly triple negative breast cancer (TNBC).

Crystal structure of ERR $\alpha$ shows that it exhibits a canonical three layered " $\alpha$ helical sandwich" fold composed of $12 \alpha$ helices (H1-H12) and two $\beta$-sheets. As shown in Fig. 1, the 

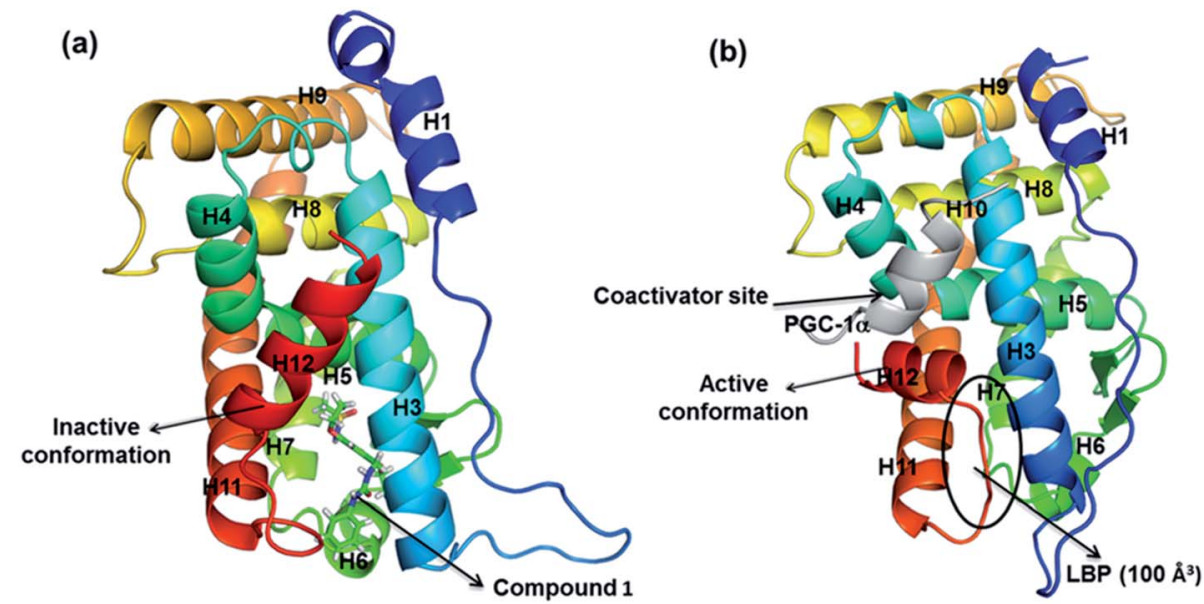

Fig. 1 (a) ERR $\alpha$ bound with compound 1 (PDB ID: 2PJL); (b) ERR $\alpha$ bound with PGC-1 $\alpha$ (PDB ID: 1XB7).

complex of ERR $\alpha$ with the inverse agonist compound 1 (inactive conformation, Fig. 1a) and ERR $\alpha$ bound with PGC- $1 \alpha$ (active apo conformation, Fig. 1b) reveal a conserved structure in the binding site region. ${ }^{13,14}$ Nevertheless, significant divergences in both $\mathrm{H} 3$ and $\mathrm{H} 12$ were observed. $\mathrm{H} 12$ is quite flexible, and upon activation $\mathrm{H} 12$ binds with coactivator normally. However, in the presence of the inverse agonist, a major conformational change takes place in the LBP where $\mathrm{H} 12$ is displaced to cap the ligand binding site, so the PGC- $1 \alpha$ is not able to combine with ERR $\alpha$ any more since the binding position is occupied by H12.

Several classes of ERR $\alpha$ inverse agonists have been reported to inhibit tumor development and progression probably by disrupting the interaction of ERR $\alpha$ with their coactivators. ${ }^{15-19}$ Among them, thiadiazoleacrylamide (XCT790) is the most potent and selective inhibitor of ERR $\alpha$, and compound 1, cyclohexylmethyl-(1- $p$-tolyl-1 $H$-indol-3-ylmethyl)-amine was the most investigated inverse agonist. X-ray crystallographic study demonstrated the interaction of compound 1 with the ligand binding pocket of ERR $\alpha^{4,17}$ Recently, a novel class of ERR $\alpha$ inverse agonists, 1-(2,5-diethoxy-benzyl)-3-phenyl-area analogues (compounds 2 and 3 ) were reported through structural optimization and ERR $\alpha$ in vitro and in vivo assays. Compound 3 shows strong inhibitory effects on the transcriptional activity of ERR $\alpha$ and could be a potent ERR $\alpha$ inverse agonist for the treatment of breast cancer. ${ }^{20}$ Structures of XCT790 and other inverse agonists, compounds 1, 2, and 3 were shown in Fig. 2.

The crystal structures of XCT790/ERR $\alpha$, compounds 2 and $3 /$ ERR $\alpha$ complexes have not been reported yet, which lags the understanding the mechanism by which the efficient inverse agonists deactivate ERR $\alpha$, e.g., the binding modes of the inverse agonists with the residues of ERR $\alpha$, and hot spot residues that play significant role in the binding, etc. To fill the gap and provide a good understanding about the binding mechanism of the inverse agonists and ERR $\alpha$, in the present work all atom molecular dynamics (MD) simulation, and free energy calculations with MM-PBSA, which are efficient tools to investigate thermodynamic properties of proteins and protein-inhibitor interactions, ${ }^{21,22}$ will be performed for the complexes consisting of inverse agonists (XCT790 and compounds 1-3) and ERR $\alpha$. The stable trajectories from MD simulations will provide detailed binding modes information on the specificity and selectivity of inverse agonists in the LBP of ERR $\alpha$. The total binding affinity will allow us to compare the binding strength of the inverse agonists with ERR $\alpha$, and analyze the contribution of different free energy components. Furthermore, per-residue basis decomposition of binding affinity was finally performed using MM-GBSA in order to quantitatively identify hot spot residues that play important roles in the binding. This study was expected to provide significant molecular and dynamic information for the design of inverse agonists that can block the ERR $\alpha$ interaction with coactivator PGC- $1 \alpha$.

\section{Materials and methods}

\subsection{Loop modeling}

The ERR $\alpha$ structure from the crystal structure of ERR $\alpha$ complexed with compound 1 (PDB ID: 2PJL) is incomplete with a few missing amino acid residues in the ranges of 309 to 317 (PDPAGPDGH) and 462 to 470 (RAGPGGGAE). A complete structure of protein is essential for accurate MD simulation studies; therefore, the missing loops in protein structure were built using ModLoop in modeling software..$^{23,24}$

\subsection{Molecular docking}

Autodock Vina was reported to have good performance on predicting binding pose and affinity, ${ }^{25}$ thus it was then used for performing docking on the basis of the above complete ERR $\alpha$, and AutoDockTools software was employed to prepare and analyze the docking results. ${ }^{26}$ Molecular structure of XCT790, compounds 2 and 3 were optimized with $\mathrm{HF} / 6-31 \mathrm{G}^{*}$ in Gaussian 09 package. ${ }^{27}$ Kollman charges, solvation parameters and polar hydrogens were added to the protein structure, and Gasteiger charge was assigned to the ligands using standard docking protocol. In docking calculations, the protein-ligand poses were ranked using an energy based scoring function. After 
<smiles>COc1cc(/C=C(\C#N)C(=O)Nc2nnc(C(F)(F)F)s2)ccc1OCc1ccc(C(F)(F)F)cc1C(F)(F)F</smiles>

XCT790<smiles>Cc1ccc(-n2cc(CNCC3CCCCC3)c3ccccc32)cc1</smiles><smiles>CCOc1cc(NS(C)(=O)=O)c(OCC)cc1CNC(=O)Nc1ccccc1</smiles>

compound 2<smiles>CCOc1cc(NS(C)(=O)=O)c(OCC)cc1CNC(=O)Nc1ccc(OCC)c(C(=O)NCc2ccc(OC)cc2)c1</smiles>

Fig. 2 The structures of the inverse agonists forestrogen-related receptor $\alpha$.

all outputs were clustered based on the root mean squared deviation (RMSD) values, the top pose conformation of docked ligand with the lowest energy is saved. For compounds $\mathbf{2}$ and $\mathbf{3}$ we have considered two binding conformations, the sulfonamide group projecting in and out of the LBP. In total, we have collected five docking complexes ERR $\alpha$-XCT790, ERR $\alpha-$ compound 2(a) and ERR $\alpha$-compound 3(a) where sulfonamide projected in the LBP, and ERR $\alpha$-compound 2(b) and ERR $\alpha$ compound 3(b) where sulfonamide projected out of the LBP.

To assess the reliability of Autodock Vina for the current systems, compound $\mathbf{1}$ was docked to the ERR-alpha of 2PJL. As shown in Fig. 3, the binding pose deviates from the crystal structure to some degree, yet the primary binding residues such as Val321, Leu324, Phe328, Phe382, Ala396, Gly397, Phe495, and Leu500 appear in the hydrophobic contact region. The deviation may be also partially resulted from missing solvent molecules. To achieve more reliable binding mode, it is necessary to preform MD in explicit solvent media.

\subsection{MD simulations}

All MD simulations were carried out using AMBER16 package $e^{28}$ with AMBERff14SB ${ }^{29}$ and general amber force field (GAFF) ${ }^{30}$ for protein and ligand molecules, respectively. In total, we have done MD simulations for seven structures: apo ERR $\alpha$, ERR $\alpha-$ XCT790, ERR $\alpha$-compound 1, ERR $\alpha$-compound 2(a), ERR $\alpha-$ compound 2(b), ERR $\alpha$-compound 3(a) and ERR $\alpha$-compound 3(b). All systems were hydrated in octahedral box extending $12 \AA$ outside the protein on all sides with explicit water molecules. Three-site TIP3P model was chosen to describe water molecules. ${ }^{31}$ For charge neutralization, the proper number of $\mathrm{Na}^{+}$ ions were placed randomly in the simulation box.
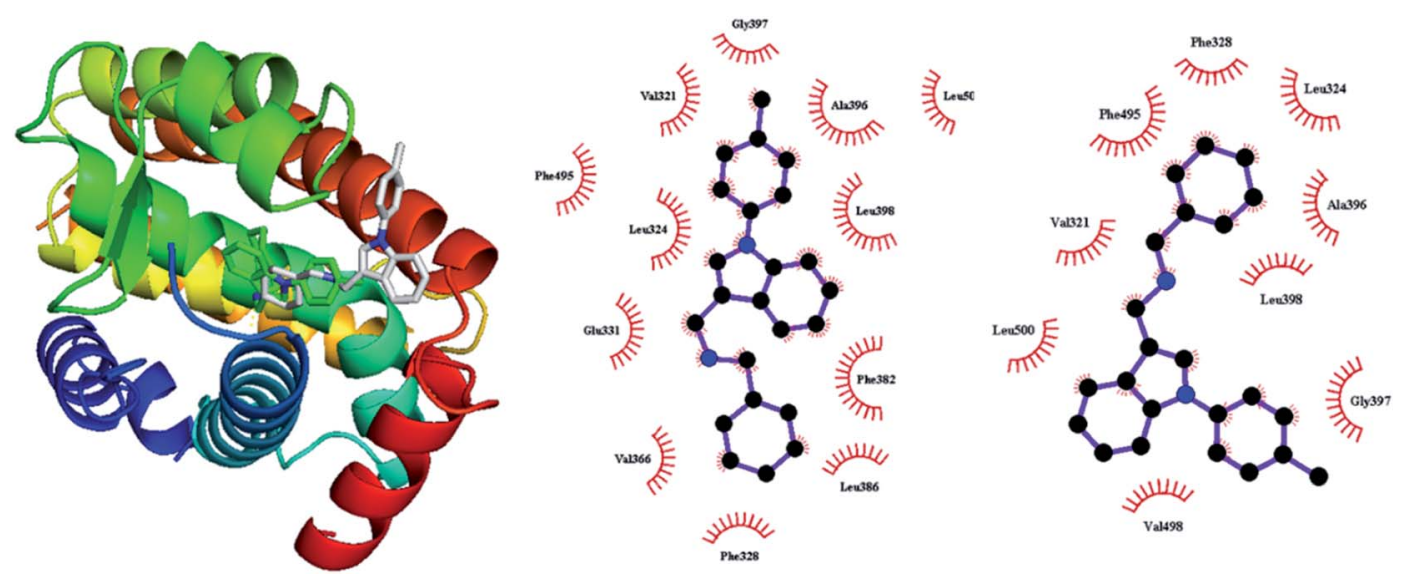

Fig. 3 The superimposed binding of compound 1 to ERR $\alpha$ from crystal (in green) and the docked (white), and 2-dimensional modes in the crystal structure (middle) and in the docked structure from Ligplot. 
All prepared systems were minimized in two steps: water molecules were firstly minimized keeping force constants over protein-ligand complexes, followed by minimization of the entire system in the second step. The first 1000 steps of energy minimization were run with steepest descent method and remaining 2000 steps with conjugate gradient method. Particle Mesh Ewald (PME) summation was used to handle the longrange coulombic interactions with a cutoff of $10 \AA$ A. Minimized systems were then slowly heated to bring system's temperature from $0 \mathrm{~K}$ to $310 \mathrm{~K}$ in NVT ensemble with time step of $0.002 \mathrm{fs}$. Systems were then equilibrated until pressure and density of systems were stabilized in NPT ensemble. For equilibration and subsequent steps, Berendsen thermostat was used in the isothermal isobaric (NPT) ensemble with target pressure of 1 bar and pressure coupling constant of 2 ps. Final production MD simulations were performed for 500 ns under NPT conditions using the GPU-supported pmemd MD module. ${ }^{32-34}$ All of the bonds involving hydrogen atoms were constrained using the SHAKE algorithm. For temperature scaling, langevin dynamics was used with a collision frequency of 2 ps.

\subsection{Binding free energy calculation}

The binding free energy of protein-ligand complexes were evaluated using MM/PBSA (Molecular Mechanics/PoissonBoltzmann Surface Area) program in AMBER16. For each complex, a total of 50 snapshots were extracted along MD trajectory from the last $100 \mathrm{~ns}$ MD simulations with an interval of 200 ps. The binding energy $(\Delta G)$ in condensed phase can be simply defined by the following equations. ${ }^{35,36}$

$$
\begin{gathered}
\Delta G=\Delta H-T \Delta S \\
\Delta H=\Delta E_{\mathrm{MM}}+\Delta G_{\mathrm{sol}}
\end{gathered}
$$

where $\Delta G$ is the binding free energy in solution that consists of the molecular mechanics energy in the gas phase $\left(\Delta E_{\mathrm{MM}}\right)$, the solvation free energy $\left(\Delta G_{\text {sol }}\right)$ and the conformational entropy effect due to the binding $(T \Delta S)$.

$$
\Delta E_{\mathrm{MM}}=\Delta E_{\mathrm{vdw}}+\Delta E_{\mathrm{ele}}
$$

where $\Delta E_{\mathrm{vdw}}$ and $\Delta E_{\text {ele }}$ correspond to the van der Waals and electrostatic interactions in gas phase, respectively.

$$
\Delta G_{\mathrm{sol}}=\Delta G_{\mathrm{pol}}+\Delta G_{\mathrm{nonpol}}
$$

where $\Delta G_{\text {pol }}$ and $\Delta G_{\text {nonpol }}$ are the polar and non-polar contributions to the solvation free energy, respectively. The $\Delta G_{\text {pol }}$ was calculated with the PBSA module, where the dielectric constant is set to 1 inside the solute and 80.0 in the solvent. The nonpolar contribution of the solvation free energy is calculated as a function of the solvent accessible surface area (SASA), as follows:

$$
\Delta G_{\mathrm{nonpol}}=\gamma(\mathrm{SAS})+\beta
$$

where, SASA was estimated using the MSMS program, with a solvent probe radius of $1.4 \AA$. The values of empirical constants $\gamma$ and $\beta$ were set to $0.000542 \mathrm{kcal} \mathrm{mol}^{-1} \AA^{-2}$ and $0.92 \mathrm{kcal} \mathrm{mol}^{-1}$, respectively. The contribution of entropy $(T \Delta S)$ to the binding free energy arising from changes in the translational, rotational and vibrational degrees of freedom was calculated by using classical statistical thermodynamics and quasi-normal mode analysis.

\subsection{Per-residue free energy decomposition analysis}

To identify the key residues responsible for the binding process, free-energy decomposition into the contribution of each residue was performed. On account of the huge demand of computational resources for PB calculations, the interaction between inverse agonists and $\operatorname{ERR} \alpha$ residues was computed using the MM-GBSA decomposition process applied in the $m m_{-} p b s a$ module in AMBER16.

\subsection{Clustering analysis}

The clustering of trajectories was performed using cpptraj module in AMBER16. The clustering was done with minimum number of points and an epsilon value of 4.0 and taking first frame as a reference. The cluster-to-cluster distance was defined as the average of all distances between individual points of two clusters according to the so-called average-linkage algorithm, which is one of the best clustering methods. ${ }^{37}$ In total, we have generated 10 clusters and considered the closest cluster conformation with reference structure.

\section{Results and discussion}

\subsection{MD trajectory and binding mode}

For ERR $\alpha$ it was difficult to predict the exact details of how the inverse agonist would bind in the LBP because of the multiple conformational changes required to create the necessary space and interactions. Similarly, it was not clear what exact consequences ligand binding would have on H12, except that it would probably be displaced from the inverse agonist position. ${ }^{4}$ To elucidate the underlying molecular mechanism and conformational changes in ERR $\alpha$ in the presence of the inverse agonists in more detail, all atom MD simulations were performed. MD simulations have been shown to be useful for assessing the binding modes observed in molecular docking calculations. The overall structural stability of ERR $\alpha$ was evaluated by RMSD of the backbone atoms. The result of RMSD obtained by MD simulations proves the stability of all complexes during the simulation and validity of docking results. Fig. 4 shows the RMSDs for the backbone atoms of the protein during $\mathrm{MD}$ simulation relative to the initial minimized structure. An analysis of the RMSD indicates that all complexes tend to converge after $35-40 \mathrm{~ns}$, suggesting that the systems became sufficiently stable through 500 ns MD simulations. The root-mean-square fluctuations (RMSF) of residues of ERR $\alpha-$ inverse agonist complexes were shown in Fig. S1. $\dagger$ Analyzing RMSF plots, similarly to the loop regions of regular proteins the modeled H2-H3 and H10-H11 loops in ERR $\alpha$ also show larger fluctuations than other regions. Apart from these two modeled loops, very minor fluctuations were observed in apo and 


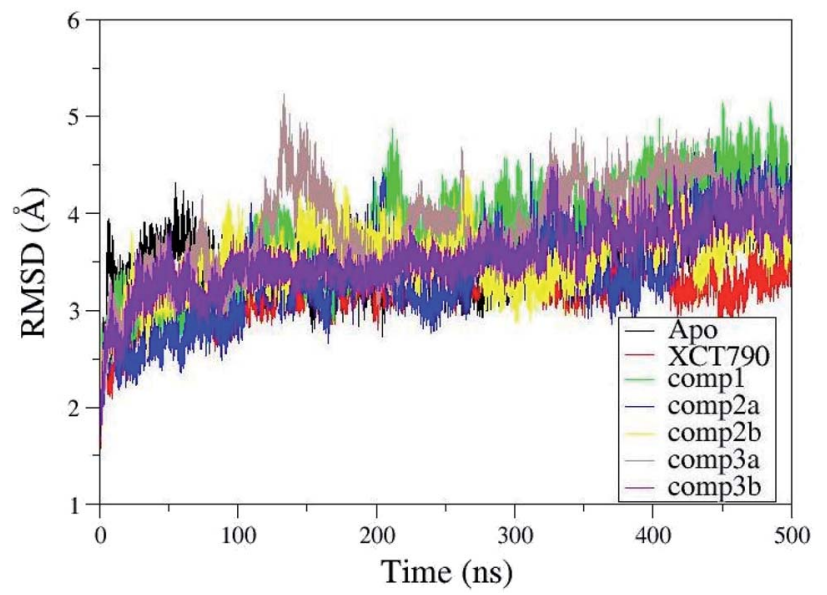

Fig. 4 Root-mean-square deviation (RMSD) plot for backbone atoms of ERR $\alpha$ and inhibitor complexes relative to their initial minimized structure as a function of time.

complexed proteins. H11-H12 loop is more stable in complexes 3(b) and XCT790 compared with apo and other complexed proteins. Interestingly, helix $\mathrm{H} 12$ is unstable in ERR $\alpha$ when complexed with compound $3(\mathbf{b})$, implicating that compound 3 strongly binds to H11-H12 loop and shifts the H12 loop to the region where PGC- $1 \alpha$ bound to ERR $\alpha$.

The detailed binding of inverse agonists to ERR $\alpha$ was analyzed for the most populated cluster of each complex in 2dimensional as well as 3-dimensional modes. 2-dimensional modes from Ligplot in ESI (Fig. S2 and S4 $\dagger$ ) show available hydrophobic contacts and hydrogen bonds of the inverse agonists with ERR $\alpha$.The following 3-dimensional modes were generated with Pymol by displaying the residues $4.0 \AA$ around the inverse agonists. Significant disruptions were revealed in protein-ligand contacts from their initial docking poses. Analyzing the top cluster of each ERR $\alpha$-inverse agonist complex, it shows that ligand binding pocket is primarily delineated (cutoff $4.0 \AA$ ) by the residues from H3, H11, H5/H6 loop and H6/H7 loop.
The binding modes of inverse agonist compound $\mathbf{1}$ in the LBP of ERR $\alpha$ are shown in Fig. 5a. The LBP of ERR $\alpha$ consists of 20 residues, most of which have hydrophobic side chains and come from H3 (Val321, Leu324, Ser325, Leu327, Phe328, Glu331), H5 (Met362, Leu365, Val366, Val369), the $\beta$-sheet (Phe382), H6 (Ala396), the H6/H7 loop (Gly397, Leu398), H7 (Leu401, Leu405), H11 (Val491, Phe495, Val498), and H11/H12 loop (Leu500). Apart from Ser325 and Gly397, the rest of the residues were also predicted by the X-ray crystal structure for the ERR $\alpha$ complex with inverse agonist compound $1 .^{4}$ The good agreement indicates that the current all-atom MD simulation is able to provide reasonable prediction for the binding of ERR $\alpha$ with relevant ligands. 2-D plot in Fig. S2(b) $\dagger$ shows strong hydrophobic interaction with six residues, Val321, Leu324, Phe328, Val369, Ala396, and Phe495.

It is reported that inhibition of ERR $\alpha$ by its inverse agonist XCT-790 can suppress the proliferation, decrease G2/M phases, and induce mitochondrial-related apoptosis of TNBC cells, ${ }^{38}$ and was considered as the most potent one so far. Fig. $5 \mathrm{~b}$ shows that in the LBP of ERR $\alpha$ XCT790 also primarily interacts with 20 amino acids from H3, H5, H6 and H11 regions, of which 19 are in common with those in the presence of compound $\mathbf{1}$ as discussed above.

The experimental study performed by Du et al., showed that compound 3 demonstrates strong inhibitory effects on the transcriptional activity of ERR $\alpha$ in human MDA-MB-231 cells in a dose dependent manner and the growth of ER-negative MDAMB-231 human breast cancer xenografts in vivo. ${ }^{20}$ For the sake of comparison the binding of compound 2 with ERR $\alpha$ was also illustrated. Three more residues exist in conformation 2a (Fig. 6) than above discussed compound 1 and XCT790, and phenyl group in the end of compound 2 interacts with more residues from H11 and H12. However, for conformation $2 \mathbf{b}$ only 15 residues were located in the LBP of EER $\alpha$. Comparing the binding modes of $\mathbf{2 a}$ and $\mathbf{2} \mathbf{b}$, it shows common hydrophobic contacts with residues Leu324, Ser325, Phe328, Val491, and Leu500 (Fig. S3†). For conformations $\mathbf{3 a}$ and $\mathbf{3 b}$ (Fig. 7) up to twenty-seven residues were observed in the LBP. The complementarity of fit in the hydrophobic regions is good in both
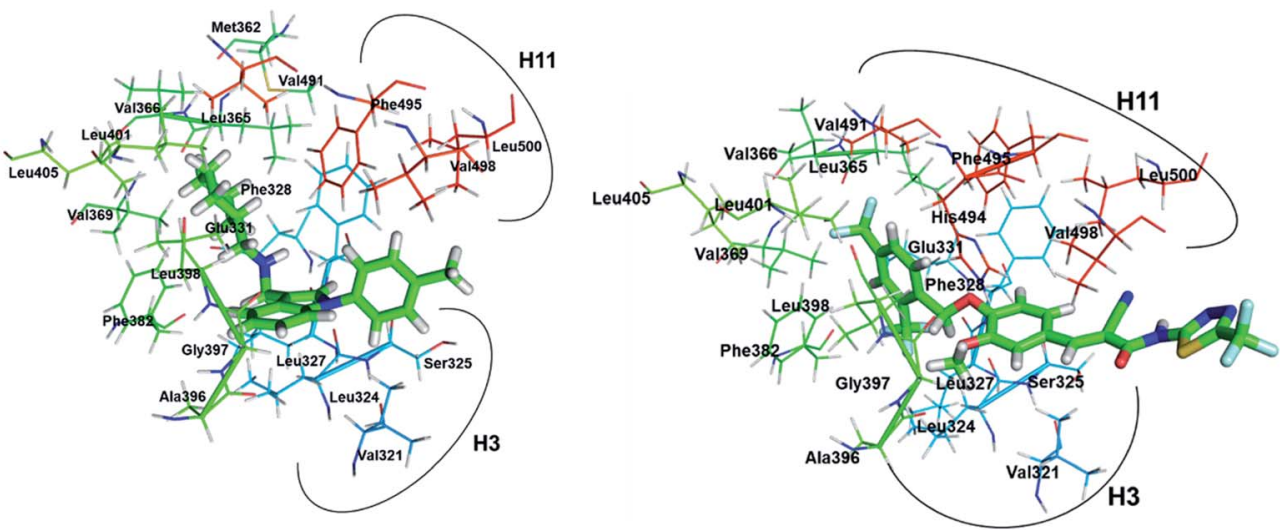

Fig. 5 Binding modes of (left) compound 1; and (right) XCT790 in the LBP of ERR $\alpha$. The ligand is shown in stick and amino acids surrounding ligand (up to $4 \AA$ ) in line representation. $\mathrm{H} 3$ and $\mathrm{H} 11$ helix regions are marked. 

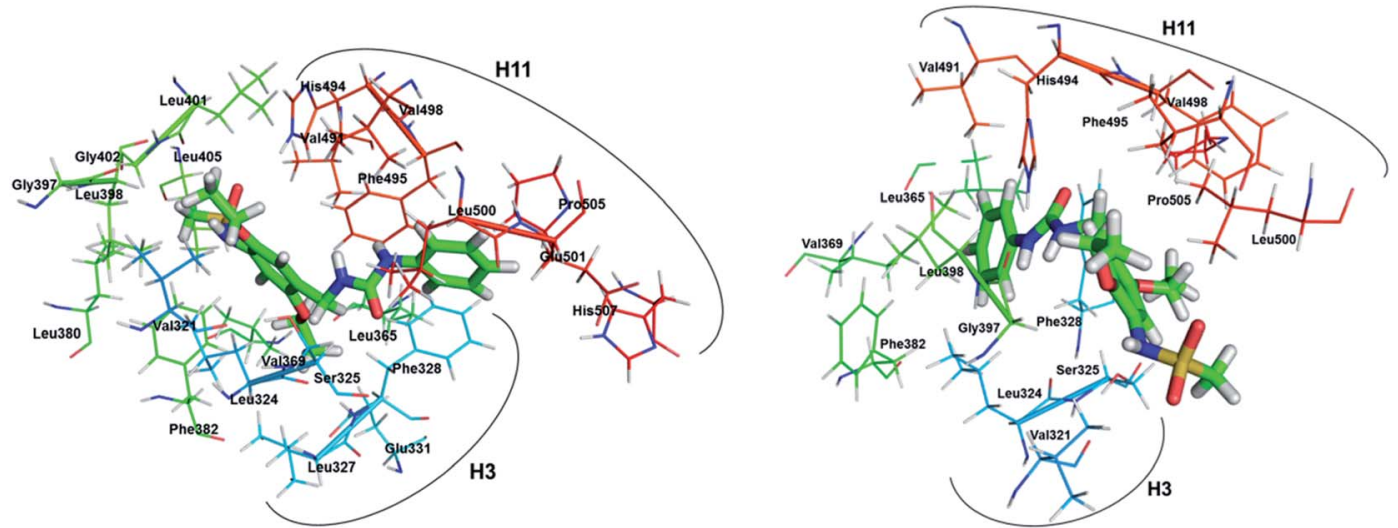

Fig. 6 Binding modes of (left) conformation 2a; (right) conformation 2b in the LBP of ERR $\alpha$. Ligands are shown in stick representation and amino acids that are surrounding ligand (up to $4 \mathrm{~A}$ ) in line representation. $\mathrm{H} 3$ and $\mathrm{H} 11$ helix regions are marked.

conformations, and its interaction is likely to contribute substantially to the binding affinity. The sulfonamide group in $3 \mathrm{a}$ forms a hydrogen bond interaction with carboxyl group of Glu331 of ERR $\alpha$ that corresponds to Glu353 of ER $\alpha$, and NH group forms hydrogen bond with Phe382 ( $\beta$ sheet).

\subsection{Binding free energy between ERR $\alpha$ and the inverse agonists}

Binding free energy calculation on the basis of stable MD simulation trajectory is the most accurate strategy to substantiate binding of compounds with favorable thermodynamics. The final $100 \mathrm{~ns}$ (400-500 ns) of each MD trajectory was used to perform MM/PBSA binding free energy calculations, and results were reported in Table 1 and shown in Fig. 8. In general, van der Waals term $\left(\Delta E_{\mathrm{vdw}}\right)$ is more favorable to the ligand binding than the electrostatic interactions $\left(\Delta E_{\text {ele }}\right)$, and nonpolar solvation energy $\left(\Delta G_{\text {nonpol }}\right)$ contributes to binding affinity by -4 to $-6 \mathrm{kcal} \mathrm{mol}^{-1}$. For the investigated inverse agonists, compound $\mathbf{3}$ (in conformations $\mathbf{3} \mathbf{a}$ and $\mathbf{3 b}$ ) has the strongest van der Waals binding $\left(-64.0\right.$ and $\left.-60.5 \mathrm{kcal} \mathrm{mol}^{-1}\right)$ followed by XCT790

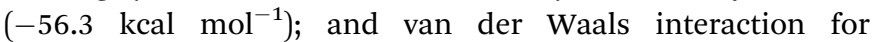
compound $1\left(\Delta E_{\mathrm{vdw}}\right.$ : $\left.-45.8 \mathrm{kcal} \mathrm{mol}^{-1}\right)$ is further weaker, yet compound 2 is the least one $\left(\sim-41.0 \mathrm{kcal} \mathrm{mol}^{-1}\right.$ in $2 \mathbf{a}$ and $\mathbf{2 b}$ conformations). The sequence of van der Waals strength for the binding of ERR $\alpha$ with the inverse agonists is roughly in line with the number of residues in the LBP analyzed above for the complexes. The binding enthalpy $\left(\Delta H=\Delta E_{\mathrm{vdw}}+\Delta G_{\text {ele }}+\Delta G_{\mathrm{pol}}+\right.$ $\Delta G_{\text {nonpol }}$, a summation of $\Delta E_{\mathrm{vdw}}, \Delta E_{\text {ele }}$, and $\Delta G_{\text {nonpol }}$ with counter contribution polar solvation energy $\left(\Delta G_{\mathrm{pol}}\right)$, has a similar trend to van der Waals term $\left(-40.0 \mathrm{kcal} \mathrm{mol}^{-1}\right.$ for compound 3, $-36.4 \mathrm{kcal} \mathrm{mol}^{-1}$ for XCT790, $-30.0 \mathrm{kcal} \mathrm{mol}^{-1}$ for compound 2, and only $-22.3 \mathrm{kcal} \mathrm{mol}^{-1}$ for compound 1).

A ligand that binds a protein becomes less mobile, and the resulting loss in configurational entropy $(-T \Delta S)$ opposes the attractive forces $(\Delta H)$ driving the binding. ${ }^{39}$ In spite of strong binding enthalpies, the penalties in binding affinity due to entropy loss $\left(-T \Delta S: \sim 20-30 \mathrm{kcal} \mathrm{mol}^{-1}\right)$ considerably weaken binding affinities $(\Delta G)$ for the inverse agonists, even bringing about a small positive one for compound 1 . The predicted trend for $\Delta G$ in Table 1 for XCT790, compound 2 and compound 3 follows the experimental $\mathrm{IC}_{50}$.

On the basis of careful comparison, Hou et al. reported that MM-GBSA has better performance than MM-PBSA to most protein-ligand systems. ${ }^{40-42}$ The binding thermodynamics from MM-GBSA was therefore included in Table 1 . The general binding affinity $\left(\Delta G_{\mathrm{GB}}\right)$ trend is the same as that from MM-PBSA
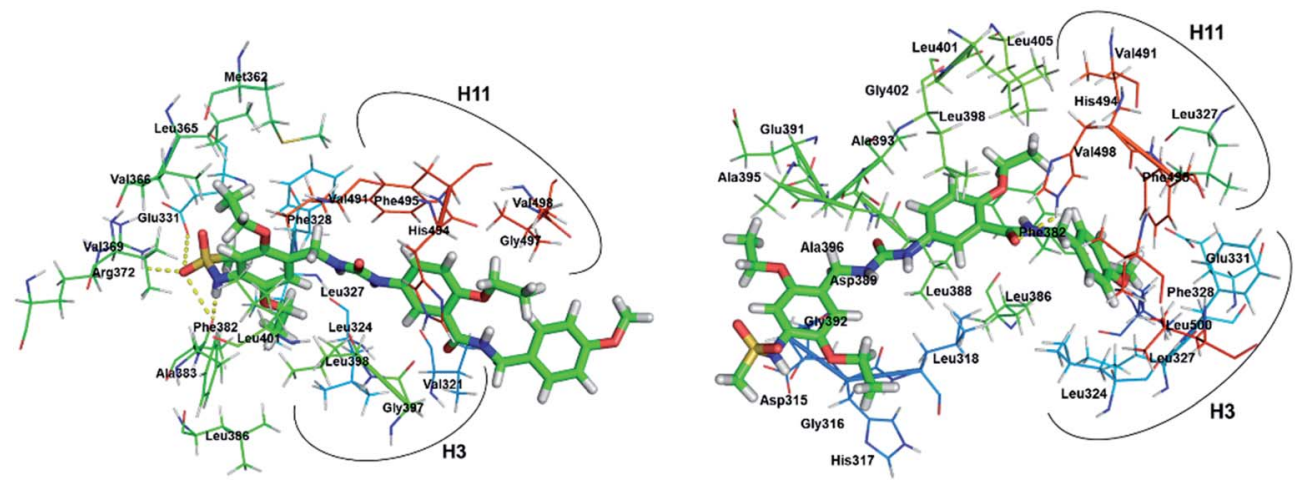

Fig. 7 Binding modes of (left) conformation 3a; (right) conformation 3b in the LBP of ERR $\alpha$. Ligands are shown in stick representation and amino acids that are surrounding ligand (up to $4 \AA$ ) in line representation. $\mathrm{H} 3$ and $\mathrm{H} 11$ helix regions are marked. 
Table 1 Binding thermodynamics of ERR $\alpha$ and inverse agonists from MM-PBSA and MM-GBSA methods. All energies are reported in kcal mol ${ }^{-1}$, and the data in the parenthesis are the standard deviation

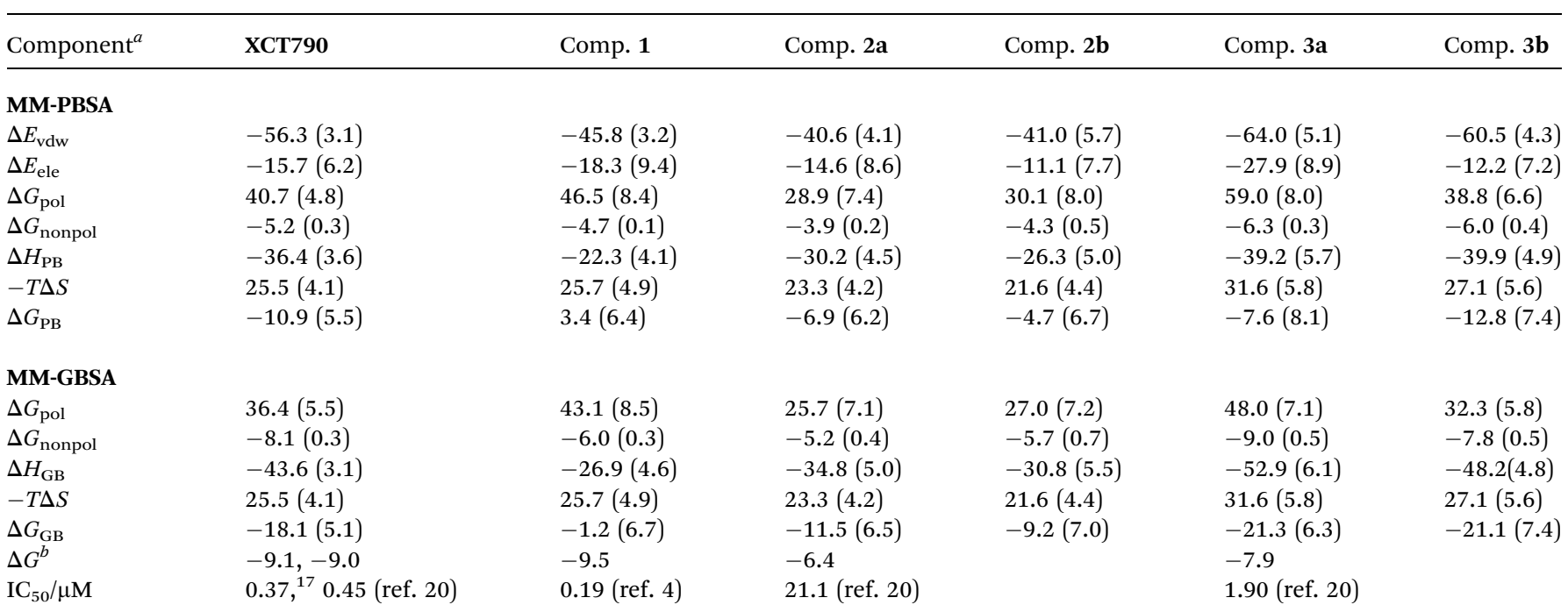

${ }^{a}$ Component: $\Delta E_{\mathrm{vdw}}$ is the van der Waals free energy; $\Delta E_{\text {ele }}$ is the electrostatic free energy; $\Delta G_{\mathrm{pol}}$ is the polar solvation energy; $\Delta G_{\text {nonpol }}$ is the nonpolar solvation energy; $\Delta H=\Delta E_{\mathrm{vdw}}+\Delta E_{\mathrm{ele}}+\Delta G_{\mathrm{pol}}+\Delta G_{\text {nonpol; }}-T \Delta S$ is the entropic contribution.; $\Delta H_{\mathrm{PB}}\left(\Delta G_{\mathrm{PB}}\right)$ and $\Delta H_{\mathrm{GB}}\left(\Delta G_{\mathrm{GB}}\right)$ were obtained from MM-GPBSA and MM-GBSA, respectively. ${ }^{b} \mathrm{IC}_{50}=K_{\mathrm{i}}^{*}\left(1+[\mathrm{S}]_{0} / K_{\mathrm{m}}\right), \Delta G=R T \ln K_{\mathrm{i}}$.

$\left(\Delta G_{\mathrm{PB}}\right)$. The predicted low solvation energy loss $\left(\Delta G_{\mathrm{pol}}+\right.$ $\Delta G_{\text {nonpol }}$ ) from GBSA method considerably enhances the binding affinity, especially for the two conformations of compound 3 up to 9-10 kcal mol ${ }^{-1}$. After taking into account the standard deviation, $\Delta G_{\mathrm{GB}}$ agrees with the experimental data better than $\Delta G_{\mathrm{PB}}$.

\subsection{Binding affinity decomposition and hot spot residues}

In order to gain further insight into $\mathrm{ERR} \alpha$-inverse agonist interaction, the contributions of binding free energy was decomposed into individual residues using MM-GBSA approach. The decomposition of free energy on per-residue consists of molecular mechanics and solvation energy but does not include entropy loss. The major residues of ERR $\alpha$ that strongly interact with the inverse agonists were shown in Fig. 9 and Table 2. In general, consistent with the above binding mode analysis these residues primarily come from three regions $\mathrm{H} 3$, $\mathrm{H} 5-\mathrm{H} 7$, and $\mathrm{H} 11-\mathrm{H} 12$.

For compound 1 five residues from H3 helix (L324, S325, and F328), H6/H7 loop (Leu398), and H11 helix (F495) contribute to the binding by $\geq 1.0 \mathrm{kcal} \mathrm{mol}^{-1}$, with a few others from $\mathrm{H} 5$ (Met362, Leu365, Val366, Val369, and F382) being in a range of 0.5-1.0 kcal mol ${ }^{-1}$. ERR $\alpha$-XCT790 complex has a rather similar binding spectrum to compound 1-ERR $\alpha$. Besides the residues (L324, S325, F328, L365, V366, V369, F382, L398, and F495) that strongly bind with compound 1 a few others such as V321 and L327 from H3, and V498 and Leu500 from H11 also show

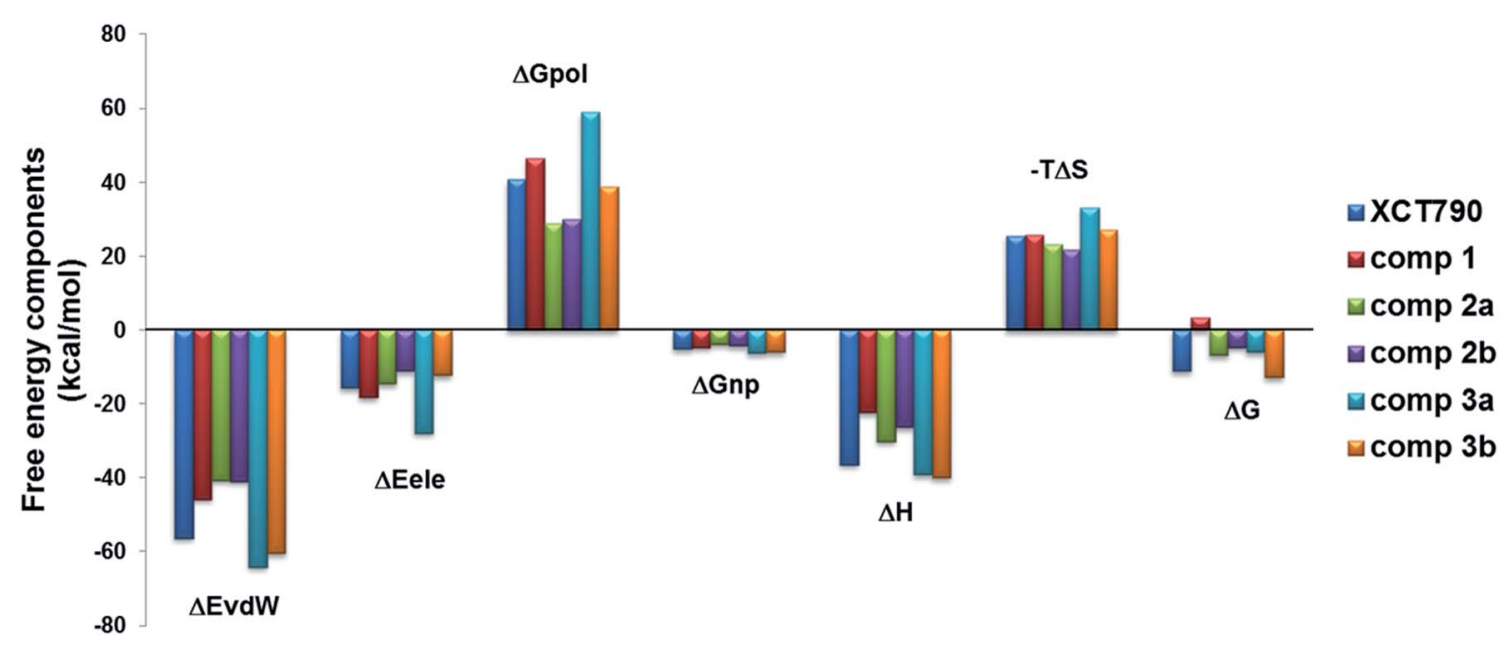

Fig. 8 Contributions of the binding free energy components of the inverse agonists to ERR $\alpha$ with MM-PBSA. 

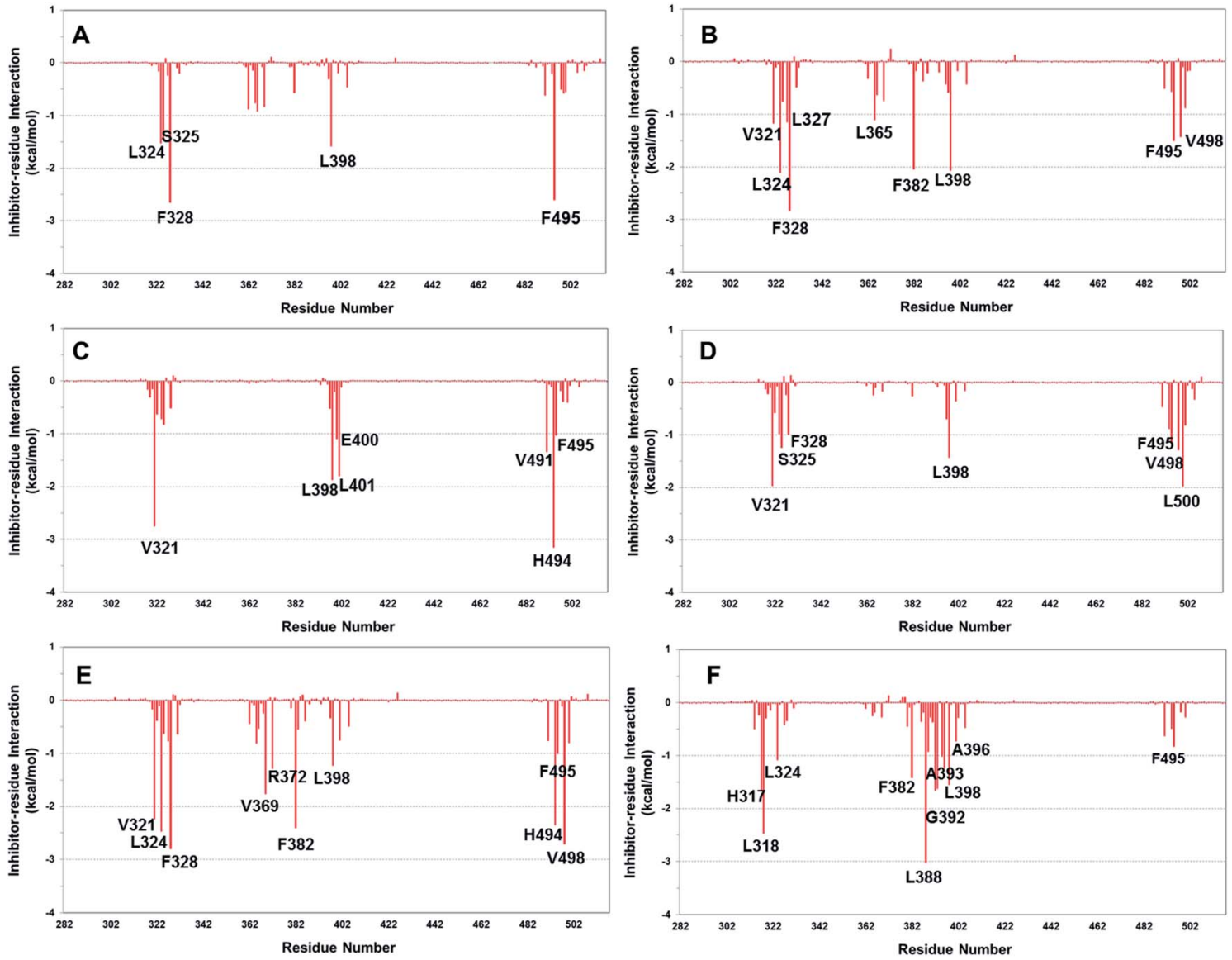

Fig. 9 Decomposition of $\Delta G$ on a per-residue basis for the protein-inhibitor complex: (A) compound 1; (B) XCT790; (C) conformation 2a; (D) conformation 2b; (E) conformation 3a (F) conformation 3b.

a significant binding by approximately $1.0 \mathrm{kcal} \mathrm{mol}^{-1}$. Met362 shows a weaker binding with XCT790 than with compound 1 $\left(-0.32\right.$ vs. $\left.-0.87 \mathrm{kcal} \mathrm{mol}^{-1}\right)$; whereas Val321 $\left(-1.17 \mathrm{kcal} \mathrm{mol}^{-1}\right)$ tends to be much stronger.

For both conformations of compound 2, in spite of strong binding with a few residues of $\mathrm{H} 3$ and $\mathrm{H} 11$ its interaction with Phe328 is much weaker than compound 1 and XCT790 $(-0.51$ vs. -2.64 and $-2.83 \mathrm{kcal} \mathrm{mol}^{-1}$ ), and it does not interact with H5-H6 either. Conformation 3a (ERR $\alpha$-compound 3) has a similar binding spectrum to those of XCT790 and compound $\mathbf{1}$; however, in conformation $\mathbf{3 b}$ of compound $\mathbf{3}$ does not interact with Phe328 and H5, which is similar to that of compound 2. It is essential for an efficient inverse agonist to strongly interact with Phe 328 of $\mathrm{H} 3$ and $\mathrm{H} 5$ so that $\mathrm{H} 3$ can displace $\mathrm{H} 5$ and $\mathrm{H} 5$ then further moves H12. Other hot spot residues with strong interaction with the EER $\alpha$ include Leu324, Phe382, Leu398, and Phe495 which considerably interact with compound 1, XCT790, as well as in conformation 3a by $1.0 \mathrm{kcal} \mathrm{mol}^{-1}$ or higher.

As shown in Fig. 10, compound 1, XCT790 and compound 3 in conformation a have a similar binding pattern in the LBP. It is speculated that LBP of ERR $\alpha$ may be bound by compound 1 to displace Phe328(H3) and Phe510(H12) that further move away H12. ${ }^{4}$ The empty cavity of the LBP in apo ERR $\alpha$ has a volume of only $\sim 100 \AA^{3}{ }^{4}$, and multiple structural adaptations are required to enable ligand binding. In apo ERR $\alpha$, the aromatic ring cluster of Phe328(H3), Phe495(H11), Phe382(H5/H6 loop) and

Table 2 Binding enthalpy decomposition results for important amino acid residues in ERR $\alpha$. All energies are reported in $\mathrm{kcal} \mathrm{mol}^{-1}$

\begin{tabular}{lllllll}
\hline Residues $^{a}$ & XCT790 & $\mathbf{1}$ & 2a & 2b & 3a & 3b \\
\hline H317 & & & & & & -1.67 \\
L318 & & & & & & -2.47 \\
Val321 & -1.17 & & -2.75 & -1.96 & -2.24 & -0.14 \\
Leu324 & -2.11 & -1.52 & -0.72 & -0.98 & -2.46 & -1.07 \\
Ser325 & -0.75 & -1.41 & -0.82 & -1.24 & -0.62 & \\
Phe328 & -2.83 & -2.64 & -0.51 & -0.99 & -2.80 & -0.34 \\
Leu365 & -1.10 & -0.76 & & & -0.81 & \\
Val366 & -0.63 & -0.92 & & & -0.53 & \\
Val369 & -0.74 & -0.83 & & & -1.76 & \\
Phe382 & -2.04 & -0.57 & & -0.25 & -2.40 & -1.41 \\
Leu398 & -2.07 & -1.58 & -1.88 & -1.43 & -1.23 & -1.55 \\
Val491 & -0.51 & -0.62 & -1.35 & -0.47 & -0.76 & -0.62 \\
His494 & -0.57 & & -3.15 & -0.88 & -2.34 & -0.49 \\
Phe495 & -1.49 & -2.60 & -1.03 & -1.15 & -1.0 & -0.83 \\
Val498 & -1.42 & -0.50 & -0.39 & -1.29 & -2.71 & -0.17 \\
Leu500 & -0.87 & -0.55 & -0.40 & -1.97 & -0.80 &
\end{tabular}

${ }^{a}$ Important amino acid residues that bind with inverse agonists. $\Delta G$ values calculated on per-residue basis. 


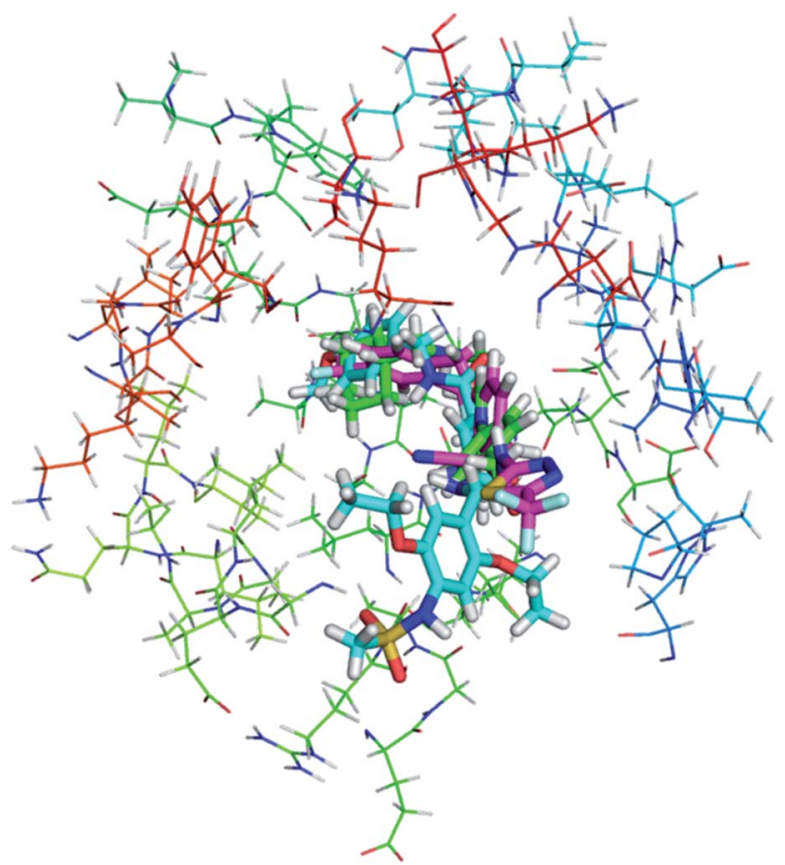

Fig. 10 Superimposition of the binding with ERR $\alpha$ in the LBP for compound 1, XCT790 compound 3 (conformation 3a).

Phe510(H12) (shown in Fig. S5 $\dagger$ ), and in particular the presence of Phe328(H3) leads to almost complete closing of the LBP with the side chain. As discussed above, XCT790, compounds 1 and 3 (in 3a conformation) considerably bind with the hot residue Phe328 with a binding enthalpy -2.6 to $-2.8 \mathrm{kcal} \mathrm{mol}^{-1}$ via $\pi-\pi$ stacking, which may trigger the displacement of other residues. Fig. S5 $\uparrow$ also shows that Phe495 and Phe328 form a hydrophobic lid on top of the ligand also through $\pi-\pi$ stacking. Such a hydrophobic lid was affected to some degree upon the binding with the inverse agonists, also reflected by the high binding enthalpy of Phe495 with the inverse agonists $(-1.0$ to $-2.6 \mathrm{kcal} \mathrm{mol}^{-1}$ ). The structure also provides the basis for rational drug design to obtain inverse agonists of ERR $\alpha$. The structure provides the basis for identification of novel inverse agonists and has broad implications for other orphan NRs including NGFI-B family, for which the LBP is completely filled with four aromatic residues conserved within the subfamily.
In order to visualize the conformational change of hot residues induced by the binding of the inverse agonists, we have superimposed apo and ligand bound ERR $\alpha$ structures for compounds 1 and 3, and XCT790 in Fig. 9. It can be seen that the hot residues superimposed in apo and ligand bound ERR $\alpha$ binding site are Val321, Leu324, Phe328, Leu365, Val369, Phe382, Leu398, Phe495, Val498, and Leu500. By superimposing apo ERR $\alpha$ with compound 1, XCT790 and compound 3a bound ERR $\alpha$ structures, it was identified that upon binding with the inverse agonists the hot residues are shifted away from the apo structure to different degree. Interestingly, Fig. 9 illustrates the considerable displacement of the hot residues, including Phe328, Phe495, and Leu500, which would move away $\mathrm{H} 12$ and in turn block the PGC-1 $\alpha$ interaction with $\mathrm{ERR} \alpha$ (Fig. 11).

\section{Conclusion}

The binding of inverse agonists such as cyclohexylmethyl-(1- $p$ tolyl-1 $H$-indol-3-ylmethyl)-amine (compound 1), thiadiazoacrylamide (XCT790), and 1-(2,5-diethoxy-benzyl)-3-phenyl-area analogues (compounds 2 and 3) with ERR $\alpha$ was comprehensively investigated using molecular docking, all atom molecular dynamics simulation, and binding free energy calculations. MD simulations show that the inverse agonists stretch downwards into the ERR $\alpha$ ligand binding pocket (LBP) primarily formed by $\mathrm{H} 3$ and H11 helices. Binding energy analysis indicates that compound 3 and XCT790 bind more tightly to ERR $\alpha$ than compounds 1 and 2, and the energy difference mainly results from the contributions of van der Waals interaction and entropy penalty. Both binding mode analysis and affinity decomposition per-residue indicate that compound 1, XCT790, and compound 3 have similar binding spectrum to ERR $\alpha$, primarily interacting with the residues of $\mathrm{H} 3, \mathrm{H} 5, \mathrm{H} 6 / \mathrm{H} 7$ loop, and $\mathrm{H} 11$ helix, while compound 2 lack of remarkable interaction with $\mathrm{H} 5$ region. The hot spot residues significantly binding to three inverse agonists include Leu324, Phe328, Phe382, Leu398, Phe495, and Leu500. It is essential for an effective inverse to strongly bind with aromatic ring cluster consisting of Phe328(H3), Phe495(H11), and Phe382(H5/H6 loop) as well as Leu500. Such understanding the binding mode of inverse agonists may provide valuable guidance for rational drug design of ERR $\alpha$ inhibitors.
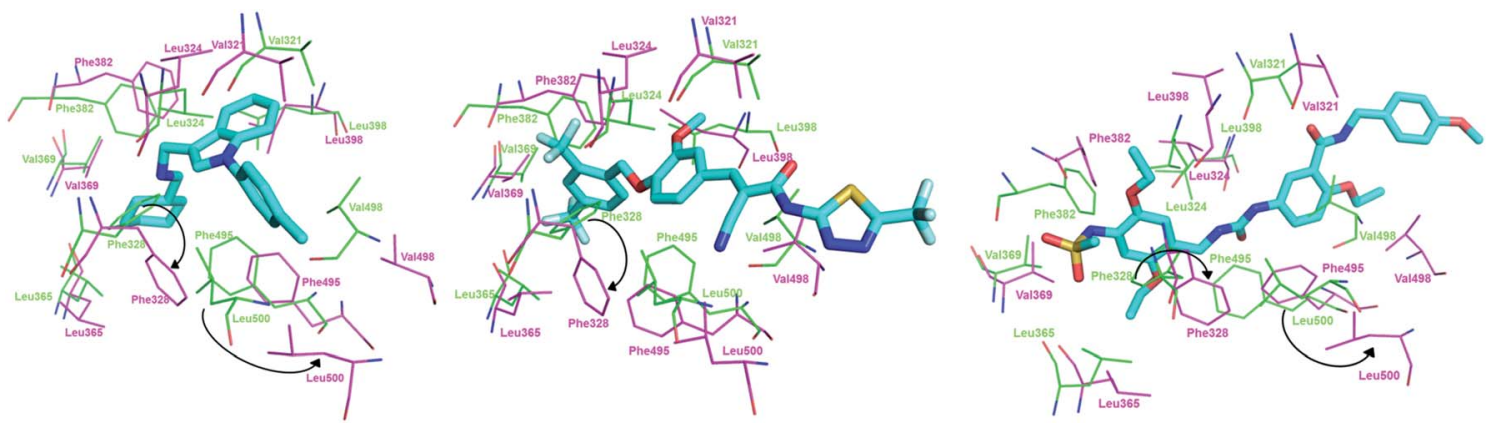

Fig. 11 Superimposition of (left) apo ERR and ERR-compound 1; (middle) apo ERR and ERR-XCT790 complex; (right) apo ERR and ERRcompound 3 (conformation 3a) complex. Residues of apo ERR $\alpha$ and bound ERR $\alpha$ are in green and magenta, respectively. The arrow shows the displacement of the hot residues, including Phe328, Phe495, and Leu500. 


\section{Conflicts of interest}

There are no conflicts to declare.

\section{Acknowledgements}

This work was supported by the National Institute of General Medical Science of the National Institute of Health (SC3GM105576 for Y. Wang), and the National Natural Science Foundation of China (81872744 for Y. Du).

\section{References}

1 V. Giguere, N. Yang, P. Segui and R. M. Evans, Nature, 1988, 331, 91-94.

2 A. M. Tremblay and V. Giguere, Nucl. Recept. Signaling, 2007, 5, e009.

3 J. M. Vanacker, K. Pettersson, J. A. Gustafsson and V. Laudet, EMBO J., 1999, 18, 4270-4279.

4 J. Kallen, R. Lattmann, R. Beerli, A. Blechschmidt, M. J. Blommers, M. Geiser, J. Ottl, J. M. Schlaeppi, A. Strauss and B. Fournier, J. Biol. Chem., 2007, 282, 2323123239.

5 B. Horard and J. M. Vanacker, J. Mol. Endocrinol., 2003, 31, 349-357.

6 T. Suzuki, Y. Miki, T. Moriya, N. Shimada, T. Ishida, H. Hirakawa, N. Ohuchi and H. Sasano, Cancer Res., 2004, 64, 4670-4676.

7 S. N. Schreiber, D. Knutti, K. Brogli, T. Uhlmann and A. Kralli, J. Biol. Chem., 2003, 278, 9013-9018.

8 V. K. Mootha, C. Handschin, D. Arlow, et al., Proc. Natl. Acad. Sci. U. S. A., 2004, 101, 6570-6575.

9 C. Y. Chang and D. P. McDonnell, Clin. Cancer Res., 2012, 18, 6089-6095.

10 J. A. Villena and A. Kralli, Trends Endocrinol. Metab., 2008, 19, 269-276.

11 C. Lynch, J. Zhao, R. Huang, N. Kanaya, L. Bernal, J. H. Hsieh, S. S. Auerbach, K. L. Witt, B. A. Merrick, S. Chen, C. T. Teng and M. Xia, Endocrinology, 2018, 159, 744-753.

12 M. Roshan-Moniri, M. Hsing, M. S. Butler, A. Cherkasov and P. S. Rennie, Cancer Treat. Rev., 2014, 40, 1137-1152.

13 J. Kallen, J. M. Schlaeppi, F. Bitsch, I. Filipuzzi, A. Schilb, V. Riou, A. Graham, A. Strauss, M. Geiser and B. Fournier, J. Biol. Chem., 2004, 279, 49330-49337.

14 F. Li, X. Sun, Y. Cai, D. Fan, W. Li, Y. Tang and G. Liu, RSC $A d v .$, 2016, 6, 94119-94127.

15 O. Lanvin, S. Bianco, N. Kersual, D. Chalbos and J. M. Vanacker, J. Biol. Chem., 2007, 282, 28328-28334.

16 H. Greschik, J. M. Wurtz, S. Sanglier, W. Bourguet, A. van Dorsselaer, D. Moras and J. P. Renaud, Mol. Cell, 2002, 9, 303-313.

17 B. B. Busch, W. C. Stevens Jr, R. Martin, P. Ordentlich, S. Zhou, D. W. Sapp, R. A. Horlick and R. Mohan, J. Med. Chem., 2004, 47, 5593-5596.
18 J. Wang, F. Fang, Z. Huang, Y. Wang and C. Wong, FEBS Lett., 2009, 583, 643-647.

19 S. Xu, X. Zhuang, X. Pan, Z. Zhang, L. Duan, Y. Liu, L. Zhang, X. Ren and K. Ding, J. Med. Chem., 2013, 56, 4631-4640.

20 Y. Du, L. Song, L. Zhang, H. Ling, Y. Zhang, H. Chen, H. Qi, X. Shi and Q. Li, Eur. J. Med. Chem., 2017, 136, 457-467.

21 K. Hart, B. Nystrom, M. Ohman and L. Nilsson, RNA, 2005, 11, 609-618.

22 Y. Miao, Y. M. Huang, R. C. Walker, J. A. McCammon and C. A. Chang, Biochemistry, 2018, 57, 1533-1541.

23 A. Fiser and A. Sali, Methods Enzymol., 2003, 374, 461-491.

24 N. Eswar, B. Webb, M. A. Marti-Renom, M. S. Madhusudhan, D. Eramian, M. Y. Shen, U. Pieper and A. Sali, Current protocols in bioinformatics, 2006, ch. 5, unit-5.6.

25 Z. Wang, H. Sun, X. Yao, D. Li, L. Xu, Y. Li, S. Tian and T. Hou, Phys. Chem. Chem. Phys., 2016, 18, 12964.

26 O. Trott and A. J. Olson, J. Comput. Chem., 2010, 31, 455-461. 27 M. J. Frisch, G. W. Trucks, H. B. Schlegel, et al., Gaussiano9 D01 edn, Gaussian Inc., Pittsburgh, PA, 2009.

28 D. A. Case, R. M. Betz and D. S. Cerutti, et al., AMBER 2016, University of California, San Francisco, 2016.

29 J. A. Maier, C. Martinez, K. Kasavajhala, L. Wickstrom, K. E. Hauser and C. Simmerling, J. Chem. Theory Comput., 2015, 11, 3696-3713.

30 J. Wang, R. M. Wolf, J. W. Caldwell, P. A. Kollman and D. A. Case, J. Comput. Chem., 2004, 25, 1157-1174.

31 W. L. Jorgensen, J. Chandrasekhar, J. D. Madura, R. W. Impey and M. L. Klein, J. Chem. Phys., 1983, 79, 926935.

32 S. Le Grand, A. W. Götz and R. C. Walker, Comput. Phys. Commun., 2013, 184, 374-380.

33 A. W. Gotz, M. J. Williamson, D. Xu, D. Poole, S. Le Grand and R. C. Walker, J. Chem. Theory Comput., 2012, 8, 15421555.

34 R. Salomon-Ferrer, A. W. Gotz, D. Poole, S. Le Grand and R. C. Walker, J. Chem. Theory Comput., 2013, 9, 3878-3888.

35 B. R. Miller 3rd, T. D. McGee Jr, J. M. Swails, N. Homeyer, H. Gohlke and A. E. Roitberg, J. Chem. Theory Comput., 2012, 8, 3314-3321.

36 S. Genheden and U. Ryde, Expert Opin. Drug Discovery, 2015, 10, 449-461.

37 J. Shao, S. W. Tanner, N. Thompson and T. E. Cheatham, J. Chem. Theory Comput., 2007, 3, 2312-2334.

38 Y. M. Wu, Z. J. Chen, G. M. Jiang, K. S. Zhang, Q. Liu, S. W. Liang, Y. Zhou, H. B. Huang, J. Du and H. S. Wang, Oncotarget, 2016, 7, 12568-12581.

39 C. E. Chang, W. Chen and M. K. Gilson, Proc. Natl. Acad. Sci. U. S. A., 2007, 104, 1534-1539.

40 L. Xu, H. Sun, Y. Li, J. Wang and T. Hou, J. Phys. Chem. B, 2013, 117, 8408-8421.

41 E. Wang, H. Sun, J. Wang, Z. Wang, H. Liu, J. Z. H. Zhang and T. Hou, Chem. Rev., 2019, 119, 9478-9508.

42 H. Sun, L. Duan, F. Chen, H. Liu, Z. Wang, P. Pan, F. Zhu, J. Z. H. Zhang and T. Hou, Phys. Chem. Chem. Phys., 2018, 20, 14450-14460. 